\title{
Maximum Entropy Approach to the Theory of Simple Fluids*
}

\author{
Chih-Yuan Tseng ${ }^{\dagger}$ and Ariel Caticha ${ }^{\ddagger}$ \\ Department of physics, University at Albany-SUNY \\ Albany, NY 12222 USA
}

\begin{abstract}
We explore the use of the method of Maximum Entropy (ME) as a technique to generate approximations. In a first use of the ME method the "exact" canonical probability distribution of a fluid is approximated by that of a fluid of hard spheres; ME is used to select an optimal value of the hard-sphere diameter. These results coincide with the results obtained using the Bogoliuvob variational method. A second more complete use of the ME method leads to a better descritption of the soft-core nature of the interatomic potential in terms of a statistical mixture of distributions corresponding to hard spheres of different diameters. As an example, the radial distribution function for a Lennard-Jones fluid (Argon) is compared with results from molecular dynamics simulations. There is a considerable improvement over the results obtained from the Bogoliuvob principle.
\end{abstract}

\section{Introduction}

The method of Maximum Entropy (ME) is designed to solve the general problem of updating from a prior probability distribution (which often happens to be a uniform distribution) to a posterior distribution when new information in the form of constraints becomes available. Of all the distributions satisfying the constraints the preferred posterior is that which is closest to the prior in the sense that it represents the least change of beliefs [1].

This suggests that ME can be used to tackle a different kind of problem. Even if a distribution is known and it accurately reflects our beliefs it may still turn out to be too complicated to be useful in practice. We may need to find a more tractable approximation. The idea is to identify a family of tractable trial distributions and then select that member which is closest to the "exact" distribution by maximizing the appropriate relative entropy. Notice

*Presented at MaxEnt 2003, the 23rd International Workshop on Bayesian Inference and Maximum Entropy Methods (August 3-8, 2003, Jackson Hole, WY, USA)

${ }^{\dagger}$ E-mail address: ct7663@csc.albany.edu

‡E-mail address: Ariel@albany.edu 
that this is not a problem that can be tackled using the more restricted version of the maximum entropy method usually known as MaxEnt. The reason is that MaxEnt only allows updating from an underlying physical measure and not from a general prior distribution.

The purpose of this paper is to develop the ME method as a technique to generate approximations. In previous work [2] we have shown that a number of well-known variational approximation schemes (the Bogoliubov variational method, the Kohn-Sham-Hohenberg density functional formalism, and even the variational method of quantum mechanics of which the Hartree-Fock method is an important special case) can be derived as special cases of the ME method. In 2 the ME method was used to develop a mean-field approximation for classical fluids in which the tractable family of distributions is obtained by replacing the interactions between molecules by an effective external mean field. The effects of the long-range attractions are described well but the short-range repulsions are badly misrepresented.

In section 2 the treatment of short-range repulsions is considerably improved by selecting as our tractable model a fluid of hard spheres [3]- [5]. The ME method is then used (section 3) to select the optimal value of the hard-sphere diameter. This is equivalent to applying the Bogoliuvob variational principle and reproduces the results obtained by Mansoori et al. [6].

An alternative approach to the study of fluids is through thermodynamic perturbation theory which yields results formally similar to the variational method. In such schemes the actual interatomic potential $u$ is replaced by $u_{0}+\delta u$, where $u_{0}$ represents the strong short-range repulsion and $\delta u$ is a long-range attraction treated as a perturbation. Remarkably, the structure of the fluid for liquid densities is dominated by the repulsive interactions. The effects of the attraction $\delta u$ are averaged over many molecules and do not appreciably affect the correlations among molecules. As a result, the first-order perturbation theory is quite accurate. At lower densities, however, higher-order corrections must be included. Since $u_{0}$ is not itself a tractable potential the usual approach is to replace it by a hard-sphere potential $u_{h s}$. Several suggestions of how to separate $u$ into $u_{0}$ and $\delta u$, of where and how to replace $u_{0}$ by $u_{h s}$, and how to choose the best hard-sphere diameter have been proposed. The most successful are the theory of Barker and Henderson 3 and, particularly, the theory of Weeks, Chandler and Andersen (WCA) 7] which succeeds in using the hard-sphere $u_{h s}$ while effectively representing the effects of the soft-core potential $u_{0}$. For a recent discussion of some of the strengths and limitations of the perturbative approach see 8 .

A clear advantage of the variational and the ME methods is that the important and yet somewhat ad hoc nature of the separation of $u$ into $u_{0}$ and $\delta u$, and of the choice of a hard-sphere diameter are eliminated. On the other hand, the variational approach fails to take the softness of the repulsive core into account, and this leads, in the end, to results that are inferior to the perturbative approaches particularly at high temperatures.

The traditional variational approach allows one to select a single optimal diameter; all non-optimal values are ruled out. But, as discussed in 1, 9, the 
ME method allows one to proceed further and quantify the extent to which non-optimal values should contribute. In this more complete use of the $\mathrm{ME}$ method, presented in section 4, the "exact" probability distribution of the fluid is approximated not by that of a gas of hard spheres with the optimal diameter but by a statistical mixture of distributions corresponding to hard spheres of different diameters. This is a rather simple and elegant way to take proper account of the fact that the actual atoms are not hard spheres but have a soft core. The full ME analysis leads to significant improvements over the variational method.

When faced with the difficulty of dealing a system described by an intractable Hamiltonian, the traditional approach has been to consider a similar albeit idealized system described by a simpler more tractable Hamiltonian. The approach we have followed here departs from this tradition: our goal is not to identify an approximately similar system but rather to identify an approximately similar probability distribution. The end result of the ME approach is a probability distribution which is a sum over distributions corresponding to different hardsphere diameters. While each term in the sum is of a form that can be associated to a real hard-sphere gas, the sum itself is not of the form $\exp -\beta H$, and cannot be interpreted as describing any physical system.

In section 5 we test our method by comparing its predictions for Argon gas with the numerical molecular dynamics simulation data obtained by Verlet [10. We find that the ME predictions for thermodynamic variables and for the radial distribution function are considerable improvements over the Bogoliuvob variational result, and are comparable to the perturbative results. Finally, our conclusions and some remarks on further improvements are presented in section 6.

\section{Approximation by hard spheres}

We consider a simple fluid composed of $N$ single atom molecules described by the Hamiltonian

$$
H\left(q_{N}\right)=\sum_{i=1}^{N} \frac{p_{i}^{2}}{2 m}+U \quad \text { with } \quad U=\sum_{i>j}^{N} u\left(r_{i j}\right),
$$

where $q_{N}=\left\{p_{i}, r_{i} ; i=1, \ldots, N\right\}$ and the many-body interactions are approximated by a pair interaction, $u\left(r_{i j}\right)$ where $r_{i j}=\left|r_{i}-r_{j}\right|$. The probability that the positions and momenta of the molecules lie within the phase space volume $d q_{N}$ is given by canonical distribution, and

$$
P\left(q_{N}\right) d q_{N}=\frac{1}{Z} e^{-\beta H\left(q_{N}\right)} d q_{N},
$$

where

$$
d q_{N}=\frac{1}{N ! h^{3 N}} \prod_{i=1}^{N} d^{3} p_{i} d^{3} r_{i} \quad \text { and } \quad Z=\int d q_{N} e^{-\beta H\left(q_{N}\right)}
$$


The difficulty, of course, is that this distribution is easy to write down but very difficult to use. We must replace $P$ by a more tractable distribution. To proceed one must recognize the two features of the interaction potential $u$ that seem to be relevant for explaining a wide variety of fluid properties; they are the strong short-distance repulsion and the weaker long-distance repulsion.

To account for the short-distance repulsion we consider the probability distribution corresponding to a gas of $N$ hard spheres of diameter $r_{d}$. The Hamiltonian is

$$
H_{h s}\left(q_{N} \mid r_{d}\right)=\sum_{i=1}^{N} \frac{p_{i}^{2}}{2 m}+U_{h s} \quad \text { with } \quad U_{h s}=\sum_{i>j}^{N} u_{h s}\left(r_{i j} \mid r_{d}\right),
$$

where

$$
u_{h s}\left(r \mid r_{d}\right)=\left\{\begin{array}{ccc}
0 & \text { for } & r \geq r_{d} \\
\infty & \text { for } & r<r_{d}
\end{array}\right.
$$

The corresponding probability distribution is

$$
P_{h s}\left(q_{N} \mid r_{d}\right)=\frac{1}{Z_{h s}} e^{-\beta H_{h s}\left(q_{N} \mid r_{d}\right)} .
$$

The partition function and the free energy $F_{h s}\left(T, V, N \mid r_{d}\right)$ are

$$
Z_{h s}=\int d q_{N} e^{-\beta H_{h s}\left(q_{N}\right)} \stackrel{\text { def }}{=} e^{-\beta F_{h s}\left(T, V, N \mid r_{d}\right)} .
$$

Two objections that can be raised to the choice of $P_{h s}$ are, first, that it does not take the long-range interactions into account; this is a point to which we will return later. Second, and this is a more serious problem, it is not clear that $P_{h s}$ is a tractable distribution at all. Indeed, the exact solution to the problem of $N$ hard spheres is not known. However, there exist analytical approximations that are in reasonably good agreement with numerical simulations. We will therefore assume that for all practical purposes $P_{h s}$ is a tractable distribution.

For hard spheres the radial correlation function can be calculated within the approximation of Percus and Yevick [11, 12] or, alternatively, from the scaled-particle theory 13. The equation of state can then be computed in two alternative ways, either from the so-called "pressure" equation, or from the "compressibility" equation but the two results do not agree. It has been found that better agreement with simulations and with virial coefficients is obtained taking an average of the two results with weights $1 / 3$ and $2 / 3$ respectively. The result is the Carnahan and Starling equation of state, $[3-[5]$

$$
\left.\frac{P V}{N k_{B} T}\right|_{h s}=\frac{1+\eta+\eta^{2}-\eta^{3}}{(1-\eta)^{3}}
$$

where $\eta=\frac{1}{6} \pi \rho r_{d}^{3}$ with $\rho=N / V$. The free energy, derived by integrating the equation of state, is

$$
F_{h s}\left(T, V, N \mid r_{d}\right)=N k_{B} T\left[-1+\ln \rho \Lambda_{T}^{3}+\frac{4 \eta-3 \eta^{2}}{(1-\eta)^{2}}\right]
$$


where $\Lambda_{T}=\left(2 \pi \hbar^{2} / m k_{B} T\right)^{1 / 2}$, and the entropy is

$$
S_{h s}\left[P_{h s}\right]=\left(\frac{\partial F_{h s}}{\partial T}\right)_{N, V}=\frac{F_{h s}}{T}+\frac{3}{2} N k_{B} .
$$

It must be remembered that these expressions are not exact. They are quite reasonable approximations for all densities up to almost crystalline densities (about $\eta \approx 0.5$ ). However, they fail to predict the face-centered-cubic phase when $\eta$ is in the range from 0.5 up the close-packing value of $\eta_{c p} \approx 0.74$.

\section{ME determination of the best hard-sphere di- ameter}

Next we address the question of which is the best $P_{h s}$. Which is the best hard-sphere diameter? According to the ME method [1, the preferred trial $P_{h s}\left(q_{N} \mid r_{d}\right)$ is that which is closest to the "exact" $P\left(q_{N}\right)$. It is found by maximizing the relative entropy,

$$
\mathcal{S}\left[P_{h s} \mid P\right]=-\int d q_{N} P_{h s}\left(q_{N} \mid r_{d}\right) \log \frac{P_{h s}\left(q_{N} \mid r_{d}\right)}{P\left(q_{N}\right)} \leq 0 .
$$

Substituting eqs.(2) and (6) we obtain

$$
\mathcal{S}\left[P_{h s} \mid P\right]=\beta\left[F-F_{h s}-\left\langle U-U_{h s}\right\rangle_{h s}\right] \leq 0,
$$

where $\langle\cdots\rangle_{h s}$ is the average computed over the hard-sphere distribution $P_{h s}\left(q_{N} \mid r_{d}\right)$. Next rewrite eq. (12) as

$$
F \leq F_{U} \stackrel{\text { def }}{=} F_{h s}+\left\langle U-U_{h s}\right\rangle_{h s}
$$

and use

$$
\left\langle U-U_{h s}\right\rangle_{h s}=\frac{1}{2} \int d^{3} r d^{3} r^{\prime}\left[u\left(r-r^{\prime}\right)-u_{h s}\left(r-r^{\prime} \mid r_{d}\right)\right] n_{h s}^{(2)}\left(r, r^{\prime}\right),
$$

where $n_{h s}^{(2)}\left(r, r^{\prime}\right)=\left\langle n^{(2)}\left(r, r^{\prime}\right)\right\rangle_{h s}$ and

$$
\hat{n}^{(2)}\left(r, r^{\prime}\right) \stackrel{\text { def }}{=} \sum_{i \neq j} \delta\left(r-r_{i}\right) \delta\left(r^{\prime}-r_{j}\right)=\hat{n}(r) \hat{n}\left(r^{\prime}\right)-\hat{n}(r) \delta\left(r-r^{\prime}\right),
$$

is the two-particle density distribution. But $u_{h s}\left(r-r^{\prime} \mid r_{d}\right)=0$ for $\left|r-r^{\prime}\right| \geq r_{d}$ while $n_{h s}^{(2)}\left(r, r^{\prime}\right)=0$ for $\left|r-r^{\prime}\right| \leq r_{d}$, therefore

$$
F_{U}=F_{h s}+\langle U\rangle_{h s} \quad \text { with } \quad\langle U\rangle_{h s}=\frac{1}{2} N \rho \int d^{3} r u(r) g_{h s}\left(r \mid r_{d}\right)
$$

where we have assumed that the fluid is homogeneous, $n_{h s}^{(2)}\left(r, r^{\prime}\right)=n_{h s}^{(2)}\left(\left|r-r^{\prime}\right|\right)$, and introduced the radial distribution function $g_{h s}$ defined by

$$
n_{h s}^{(2)}(r) \stackrel{\text { def }}{=} \rho^{2} g_{h s}\left(r \mid r_{d}\right) .
$$


Maximizing $\mathcal{S}\left[P_{h s} \mid P\right]$ is equivalent to minimizing $F_{U}$ over all diameters $r_{d}$. Thus, the variational approximation to the free energy is

$$
F(T, V, N) \approx F_{U}\left(T, V, N \mid r_{m}\right) \stackrel{\text { def }}{=} \min _{r_{d}} F_{U}\left(T, V, N \mid r_{d}\right),
$$

where $r_{m}$ is the optimal diameter. Notice that the approximation does not consist of merely replacing the free energy $F$ by a hard-sphere free energy $F_{h s}$ which does not include the effects of long range attraction; $F$ is approximated by $F_{U}\left(r_{m}\right)$ which includes attraction effects through the second term in eq. (16). This addresses the first of the two objections mentioned earlier. Indeed, the real fluid with interactions given by $u$ is not being replaced by a hard-sphere fluid with interactions given by $u_{h s}$; it is just the probability distribution that is being replaced in this way. The internal energy is approximated by $\langle H\rangle_{h s}=$ $\frac{3}{2} N k_{B} T+\langle U\rangle_{h s}$ and not by $\left\langle H_{h s}\right\rangle_{h s}=\frac{3}{2} N k_{B} T$.

To complete the solution one needs the radial distribution function $g_{h s}\left(r \mid r_{d}\right)$. A numerical solution by Throop and Bearman is tabulated in 14. Alternatively it can be written in terms of the Laplace transform of $r g_{h s}\left(r \mid r_{d}\right)$ [12],

$$
G(s)=\int_{0}^{\infty} d x x g_{h s}\left(x r_{d} \mid r_{d}\right) e^{-s x}=\frac{s L(s)}{12 \eta\left[L(s)+S(s) e^{s}\right]}
$$

where $x$ is a dimensionless variable $x=r / r_{d}$,

$$
L(s)=12 \eta\left[\left(1+\frac{1}{2} \eta\right) s+(1+2 \eta)\right],
$$

and

$$
S(s)=(1-\eta)^{2} s^{3}+6 \eta(1-\eta) s^{2}+18 \eta^{2} s-12 \eta(1+2 \eta) .
$$

To make use of this solution it is convenient to write $\langle U\rangle_{h s}$ in terms of $V(s)$, the inverse Laplace transform of $r u(r)$,

$$
x u\left(x r_{d}\right)=\int_{0}^{\infty} d s V(s) e^{-s x},
$$

which gives

$$
\langle U\rangle_{h s}=12 N \eta \int_{0}^{\infty} d s V(s) G(s),
$$

For a Lennard-Jones potential,

$$
u(r)=4 \varepsilon\left[\left(\frac{\sigma}{r}\right)^{12}-\left(\frac{\sigma}{r}\right)^{6}\right]
$$

we have

$$
V(s)=4 \varepsilon\left[\left(\frac{\sigma}{r_{d}}\right)^{12} \frac{s^{10}}{10 !}-\left(\frac{\sigma}{r_{d}}\right)^{6} \frac{s^{4}}{4 !}\right] .
$$


Finally, it remains to minimize $F_{U}$ in eq. (16). This is done numerically; an explicit example for Argon is calculated in section 5.

As mentioned in the Introduction a problem with the approach outlined above is that it fails to take the softness of the repulsive core into account. This flaw is manifested in a less satisfactory prediction of thermodynamic variables at high temperatures, and also, as will be shown by the numerical calculations in section 4 , in a poor prediction of the radial distribution function. However, as we shall see in the following sections, the ME method does not stop at the mere selection of the best diameter. A more complete ME analysis offers significant improvements over the variational method.

\section{A more complete ME analysis}

The ME method as pursued in the last section has led us to determine an optimal value of the hard-sphere diameter. Next we ask to what extent do we believe that the correct selection should be $r_{d}=r_{m}$ rather than $r_{d}=r_{m}+\delta r$. To what extent are values $\delta r \neq 0$ ruled out by the ME method? [1, 9] This question is an inquiry about the probability of $r_{d}, P_{d}\left(r_{d}\right)$. Thus, we are uncertain not just about $q_{N}$ given $r_{d}$, but also about the right $r_{d}$ and what we actually seek is the joint probability of $q_{N}$ and $r_{d}, P_{J}\left(q_{N}, r_{d}\right)$. Once this joint distribution is obtained our best assessment of the distribution of $q_{N}$ should be given by the marginal over $r_{d}$,

$$
\bar{P}_{h s}\left(q_{N}\right) \stackrel{\text { def }}{=} \int d r_{d} P_{J}\left(q_{N}, r_{d}\right)=\int d r_{d} P_{d}\left(r_{d}\right) P_{h s}\left(q_{N} \mid r_{d}\right)
$$

By recognizing that diameters other than $r_{m}$ are not ruled out and that a more honest representation is an average over all hard-sphere diameters we are effectively replacing the hard spheres by a soft-core potential.

Next, we make a second use of the ME method to obtain $P_{J}\left(q_{N}, r_{d}\right)$. Of all distributions within the family $P_{J}\left(q_{N}, r_{d}\right)=P_{d}\left(r_{d}\right) P_{h s}\left(q_{N} \mid r_{d}\right)$ we seek that which is closest to the prior $m\left(q_{N}, r_{d}\right)$ over the space $\left\{q_{N}, r_{d}\right\}$. But what is $m\left(q_{N}, r_{d}\right)$ ? We want the marginal distribution $\bar{P}_{h s}\left(q_{N}\right)$, eq.(26), as close as possible to the "exact" distribution $P\left(q_{N}\right)$ in eq.(2). This is achieved if we set $m\left(q_{N}, r_{d}\right)=P\left(q_{N}\right) \mu\left(r_{d}\right)$. Furthermore, we can assign $\mu\left(r_{d}\right)$ noting that $r_{d}$ is not a physical quantity, its only meaning derives from being a parameter appearing in the distribution $P_{h s}\left(q_{N} \mid r_{d}\right)$. Then there is a unique natural distance $d \ell^{2}=\gamma\left(r_{d}\right) d r_{d}^{2}$ in the space of $r_{d}$ s which is given by the Fisher-Rao metric,

$$
\gamma\left(r_{d}\right)=\int d q_{N} P_{h s}\left(q_{N} \mid r_{d}\right)\left(\frac{\partial \log P_{h s}\left(q_{N} \mid r_{d}\right)}{\partial r_{d}}\right)^{2} .
$$

Therefore $\mu\left(r_{d}\right)=\gamma^{1 / 2}\left(r_{d}\right)$. Therefore the joint $P_{J}\left(q_{N}, r_{d}\right)$, or equivalently the diameter distribution $P_{d}\left(r_{d}\right)$, is determined by maximizing the entropy

$$
\sigma\left[P_{d}\right]=-\int d q_{N} d r_{d} P_{d}\left(r_{d}\right) P_{h s}\left(q_{N} \mid r_{d}\right) \log \frac{P_{d}\left(r_{d}\right) P_{h s}\left(q_{N} \mid r_{d}\right)}{\gamma^{1 / 2}\left(r_{d}\right) P\left(q_{N}\right)} .
$$


For sake of simplicity, one can reorginize this entropy to

$$
\sigma\left[P_{d}\right]=-\int d r_{d} P_{d}\left(r_{d}\right) \log \frac{P_{d}\left(r_{d}\right)}{\gamma^{1 / 2}\left(r_{d}\right)}+\int d r_{d} P_{d}\left(r_{d}\right) \mathcal{S}\left[P_{h s} \mid P\right],
$$

where $\mathcal{S}\left[P_{h s} \mid P\right]$ is given eqs. (12) and (16),

$$
\mathcal{S}\left[P_{h s} \mid P\right]=\beta\left(F-F_{U}\right)=\beta\left(F-F_{h s}-\langle U\rangle_{h s}\right) .
$$

Maximizing $\sigma\left[P_{d}\right]$ over variations $\delta P_{d}$ subject to a normalization constraint gives,

$$
P_{d}\left(r_{d}\right) d r_{d}=\frac{e^{\mathcal{S}\left[P_{h s} \mid P\right]}}{\zeta} \gamma^{1 / 2}\left(r_{d}\right) d r_{d}=\frac{e^{-\beta F_{U}}}{\zeta_{U}} \gamma^{1 / 2}\left(r_{d}\right) d r_{d},
$$

where the partition functions $\zeta$ and $\zeta_{U}$ are given by

$$
\zeta=e^{\beta F} \zeta_{U} \quad \text { with } \quad \zeta_{U}=\int d r_{d} \gamma^{1 / 2}\left(r_{d}\right) e^{-\beta F_{U}}
$$

The remaining problem in the above equations is the calculation of the FisherRao measure $\gamma^{1 / 2}$ and this is conveniently done by considering the entropy of $P_{h s}\left(q_{N} \mid r_{d}^{\prime}\right)$ relative to $P_{h s}\left(q_{N} \mid r_{d}\right)$,

$$
\mathcal{S}\left[P_{h s}\left(q_{N} \mid r_{d}^{\prime}\right) \mid P_{h s}\left(q_{N} \mid r_{d}\right)\right]=-\int d q_{N} P_{h s}\left(q_{N} \mid r_{d}^{\prime}\right) \log \frac{P_{h s}\left(q_{N} \mid r_{d}^{\prime}\right)}{P_{h s}\left(q_{N} \mid r_{d}\right)} .
$$

A straightforward differentiation shows that

$$
-\left.\frac{\partial^{2} \mathcal{S}\left[P_{h s}\left(q_{N} \mid r_{d}^{\prime}\right) \mid P_{h s}\left(q_{N} \mid r_{d}\right)\right]}{\partial r_{d}^{\prime 2}}\right|_{r_{d}^{\prime}=r_{d}}=\gamma\left(r_{d}\right) .
$$

Substituting the distributions $P_{h s}\left(q_{N} \mid r_{d}^{\prime}\right)$ and $P_{h s}\left(q_{N} \mid r_{d}\right)$ into eq.(33) gives

$\mathcal{S}\left[P_{h s}\left(q_{N} \mid r_{d}^{\prime}\right) \mid P_{h s}\left(q_{N} \mid r_{d}\right)\right]=\beta\left[F_{h s}\left(r_{d}\right)-F_{h s}\left(r_{d}^{\prime}\right)-\left\langle U_{h s}\left(r_{d}\right)\right\rangle_{r_{d}^{\prime}}+\left\langle U_{h s}\left(r_{d}^{\prime}\right)\right\rangle_{r_{d}^{\prime}}\right]$,

where $\langle\cdots\rangle_{r_{d}^{\prime}}$ is the average over $P_{h s}\left(q_{N} \mid r_{d}^{\prime}\right)$. As we argued above eq.(16) the expectation of the potential energy $\left\langle U_{h s}\left(r_{d}^{\prime}\right)\right\rangle_{r_{d}^{\prime}}$ vanishes because the intermolecular potential $u\left(r \mid r_{d}^{\prime}\right)$ is zero in the region $r>r_{d}^{\prime}$ where the radial distribution function $g_{h s}\left(r \mid r_{d}^{\prime}\right)$ is not zero. A similar argument shows that $\left\langle U_{h s}\left(r_{d}\right)\right\rangle_{r_{d}^{\prime}}=0$ when $r_{d}^{\prime} \geq r_{d}$. However, when $r_{d}^{\prime} \leq r_{d}$ the expectation $\left\langle U_{h s}\left(r_{d}\right)\right\rangle_{r_{d}^{\prime}}$ diverges, $\mathcal{S}$ is not defined and eq. (34) is not applicable. We can argue our way out of this quandary by pointing out that the divergence is a consequence of the unphysical idealization involved in taking a hard-sphere potential seriously. For more realistic continuous potentials the distance between $r_{d}^{\prime}=r_{d}+d r_{d}$ and $r_{d}$ is the same as the distance between $r_{d}^{\prime}=r_{d}-d r_{d}$ and $r_{d}$. We can then always choose 
$r_{d}^{\prime} \geq r_{d}$ and define $\gamma\left(r_{d}\right)$ in eq.(34) as the limit $r_{d}^{\prime}=r_{d}+0^{+}$. Then, using eq.(9), we have

$$
\gamma\left(r_{d}\right)=\left.\beta \frac{\partial^{2} F_{h s}\left(r_{d}^{\prime}\right)}{\partial r_{d}^{\prime 2}}\right|_{r_{d}^{\prime}=r_{d}+0^{+}}=N \pi \rho r_{d} \frac{4+9 \eta-4 \eta^{2}}{(1-\eta)^{4}}
$$

To summarize, the distribution of diameters $P_{d}\left(r_{d}\right)$ is given by eq. (31) with $F_{U}$ given by eqs. (16, 20 23) and $\gamma$ given by (36). Our best approximation to the "exact" $P\left(q_{N}\right)$ is the $\bar{P}_{h s}\left(q_{N}\right)$ given in eq.(26). The corresponding best approximation to the radial distribution function is

$$
\bar{g}_{h s}(r)=\int d r_{d} P_{d}\left(r_{d}\right) g_{h s}\left(r \mid r_{d}\right) .
$$

When intermolecular interactions are given by two-body potentials the equation of state, the free energy, the internal energy, and other thermodynamic functions can be expressed in terms of the radial distribution function. Therefore an improved $g(r)$ leads to improved estimates for all other quantities.

However, there is a problem. For large $N$ the distribution $P_{d}\left(r_{d}\right) \sim \exp -\beta F_{U}$ is very sharply peaked about the maximum attained at the optimal diameter $r_{m}$ because $F_{U}$ is an extensive quantity $F_{U} \propto N$. This result must be interpreted with care: when choosing a single optimal diameter for a macroscopic fluid sample we find that the ME confers overwhelming probability to the optimal value. This is not surprising. The same thing happens when we calculate the global temperature or density of a macroscopic sample: the ME method predicts that fluctuations about the expected value are utterly negligible. And yet fluctuations can be important. For example, for smaller fluid samples, or when we consider the local behavior of the fluid, fluctuations are not merely observable but can be large. Local fluctuations can be appreciable while global fluctuations remain negligible.

The question then, is whether these local fluctuations are relevant to the particular quantities we want to calculate. We argue that they are. The radial distribution function $g(r)$ is the crucial quantity from which all other thermodynamic variables are computed. But from its very definition $-g(r)$ is the probability that given one atom at a given fixed point, another atom will be found at a distance $r$ - it is clear that $g(r)$ refers to purely local behavior and should be affected by local fluctuations. To the extent that the optimal $r_{m}$ depends on temperature and density we expect that local temperature and/or density fluctuations would induce local diameter fluctuations as well.

It is important to note that unlike density fluctuations, the local diameter fluctuations are not real. They cannot be: the hard spheres do not exist except in our minds. The diameter fluctuations are merely a representation of our uncertainty about which $r_{d}$ to choose.

The extended analysis in this section does not yet allow us to pursue the question of local fluctuations in a satisfactory manner. For the purpose of this paper, however, we can quickly estimate the effects of local fluctuations by assuming that the effective number of atoms $N_{\text {eff }}$ that are locally relevant to 
the calculation of $g(r)$ is smaller than $N$. We deal with an effectively smaller fluid sample. The actual calculation of $N_{\text {eff }}$ will be pursued elsewhere; it is not difficult to see that the ME method itself still applies [9], all that is needed is a broader family of trial distributions.

Next we apply the extended ME method developed above to fluid Argon.

\section{An example: Argon}

One of the difficulties in testing theories about fluids against experimental data is that it is not easy to see whether discrepancies are to be blamed to a faulty approximation or to a wrong intermolecular potential. This is why it is theories are normally tested against molecular dynamics numerical simulations where there is control over the intermolecular potential. In this section we compare ME results against simulation results [10] for fluid of monatomic molecules interacting through a Lennard-Jones potential (24). The parameters $\varepsilon$ and $\sigma$ (the depth of the well and the radius of the repulsive core, $u(\sigma)=0$ ) are chosen to model Argon: $\epsilon=10^{-2} \mathrm{eV}$ and $\sigma=3.405 \AA$.

Figure 1 1 shows the free energy $F_{U} / N k_{B} T$ as a function of hard-sphere diameter $r_{d}$ for Argon at a density of $\rho \sigma^{3}=0.65$ for different temperatures, and fig 2 shows $F_{U} / N k_{B} T$ as a function of $r_{d}$ for several densities at fixed $T=107.2 \mathrm{~K}$. Since the critical point for Argon is $T_{c}=150.69 \mathrm{~K}$ and density $\rho \sigma^{3}=0.33$ all these curves lie well within the liquid phase. The increase of $F_{U} / N k_{B} T$ for low values of $r_{d}$ is due to short range repulsion described by $F_{h s} / N k_{B} T$ while the increase for large $r_{d}$ is due to the long range attraction described by $\langle U\rangle_{h s} / N k_{B} T$.

The best $r_{d}$ is that which minimizes $F_{U}$ and depends both on temperature and density. The best diameter decreases as the temperature increases: atoms with higher energy can penetrate deeper into the repulsive core. The dependence with density is less pronounced.

Next we study the distribution of diameters, eq. (31). As discussed earlier for large $N$ the distribution $P_{d}\left(r_{d}\right) \sim \exp -\beta F_{U}$ is sharply peaked about the optimal diameter $r_{m}$. But we argued that the effective number of particles that are relevant to the local behavior is smaller $N_{\text {eff }}$. In Fig 3 we plot the distribution $P_{d}\left(r_{d}\right)$ for different temperatures, for a fixed fluid density of $\rho \sigma^{3}=0.65$, and for an arbitrarily chosen fixed $N_{\text {eff }}=13500$. Notice again that the distribution shifts to lower diameters as the temperature increases. Notice also that at lower temperatures the distribution becomes narrower. This means that the effects of the softness of the repulsive core are less important; that a hard-sphere approximation is better at low temperatures [3].

Finally in fig.(4) we show a comparison of the radial distribution functions computed in three ways. The dotted line is $g_{h s}\left(r \mid r_{m}\right)$ for the hard-sphere fluid with optimal diameter $r_{m}$. This curve, calculated from the numerical data tabulated in [14, is also the result of the variational method and coincides with the ME result for a macroscopically large $N_{\text {eff }}=N$. The dashed line is Verlet's molecular dynamics simulation The continuous line is the averaged $\bar{g}_{h s}(r)$ of 


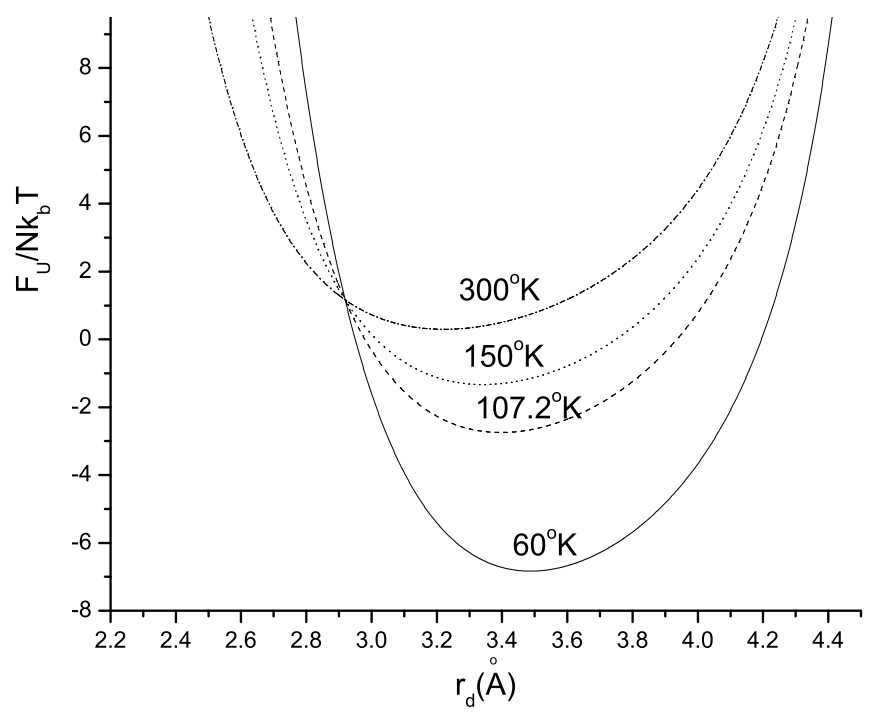

Figure 1: The free energy $F_{U}$ as a function of hard-sphere diameter $r_{d}$ for Argon at a density of $\rho \sigma^{3}=0.65$ for different temperatures. The best $r_{d}$ is that which minimizes $F_{U}$.

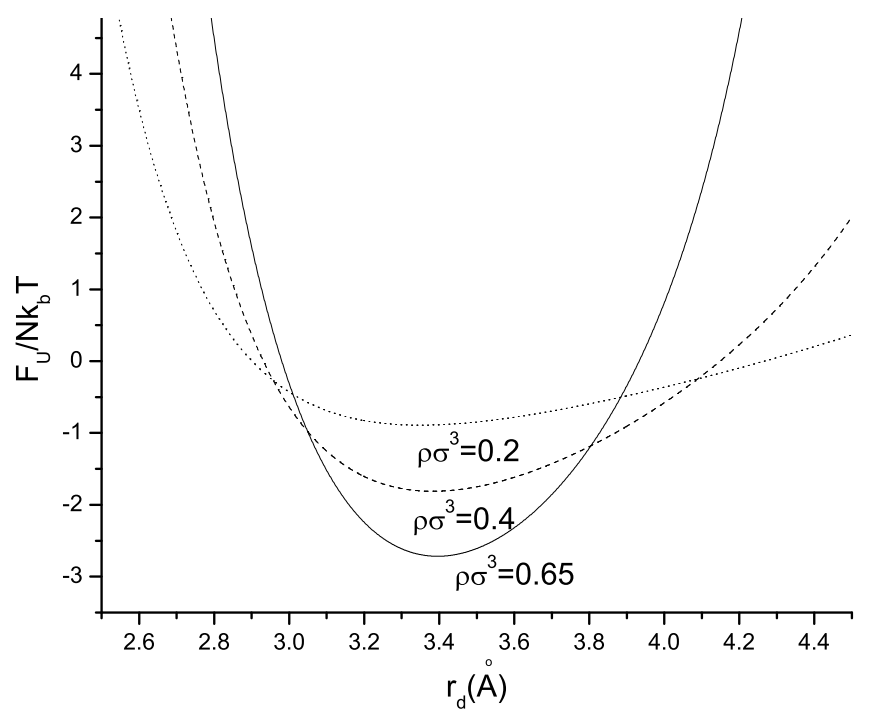

Figure 2: The free energy $F_{U}$ as a function of hard-sphere diameter $r_{d}$ for Argon at $T=107.82 \mathrm{~K}$ for different densities. The best $r_{d}$ is that which minimizes $F_{U}$. 


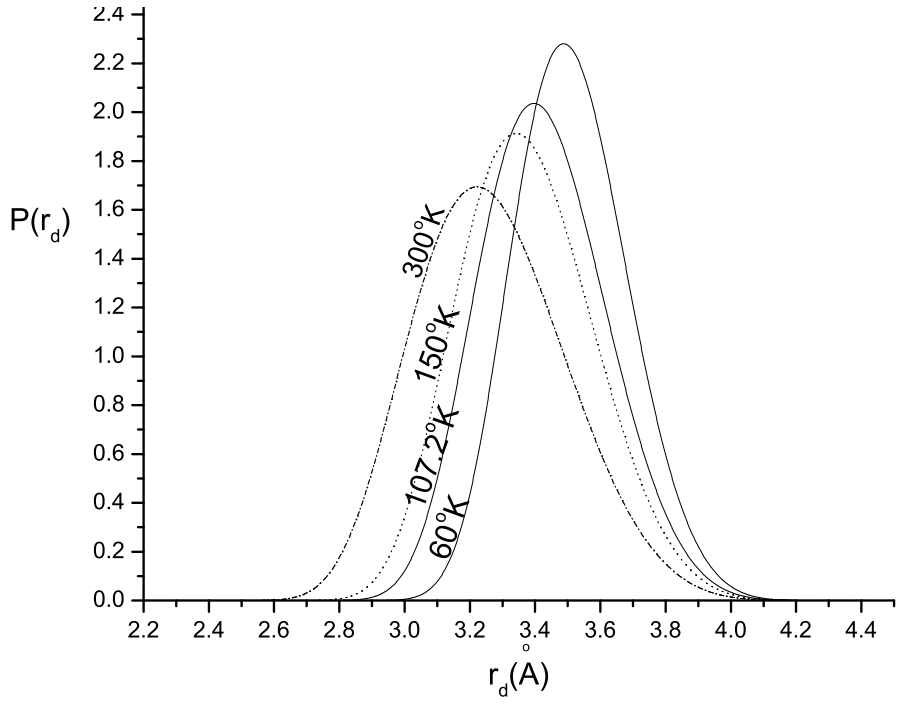

Figure 3: The distribution of hard-sphere diameters $r_{d}$ for Argon for several temperatures at density $\rho \sigma^{3}=0.65$. The size of fluid was arbitrarily chosen as $N_{\text {eff }}=13500$.

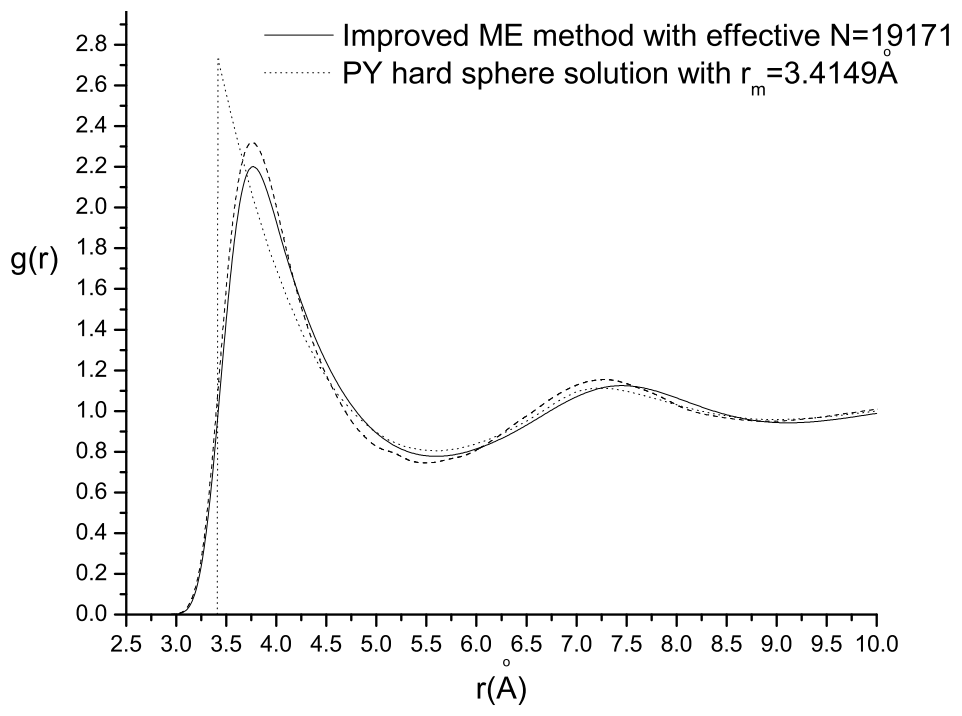

Figure 4: The radial distribution function for (a) the hard-sphere fluid with optimal diameter $r_{m}$; (b) Verlet's molecular dynamics simulation; and (c) the improved ME analysis, for Argon at density $\rho \sigma^{3}=0.65$, temperature $T=$ $107.82 \mathrm{~K}$, and effective particle number $N_{\text {eff }}=19171$. 
the more extended ME analysis. The calculation is for a density $\rho \sigma^{3}=0.65$, temperature $T=107.82 \mathrm{~K}$, and the value of effective particle number $N_{\text {eff }}=$ 19171 was adjusted to achieve the best fit. The agreement between the ME curve and Verlet's data is remarkably good. The vast improvement over the simpler variational method calculation is clear.

One might be tempted to dismiss this achievement as due to the adjustment of the parameter $N_{\text {eff }}$ but this is not quite fair: there is a single free parameter and the functional form of the whole curve $\bar{g}_{h s}(r)$ in eq.(37) is correctly reproduced. We should point out, for example, that the Fisher-Rao measure $\gamma^{1 / 2}\left(r_{d}\right)$ plays a crucial role. Omitting the factor $\gamma^{1 / 2}\left(r_{d}\right)$ from the distribution $P_{d}\left(r_{d}\right)$ would have led to a much less satisfactory fit regardless of the choice of $N_{\text {eff }}$.

\section{Conclusion}

The main goal of this paper has been to show that the ME method can be used to generate approximations in a way that generalizes the Bogoliuvob variational principle. This addresses a range of applications that lie beyond the scope of the traditional MaxEnt.

We showed that rather than approximating the real fluid by a fictitious one, it is better to approximate the "exact" probability distribution by a statistical mixture of distributions corresponding to hard spheres of different diameters which allows a better description of the soft core of the short-range repulsive potential.

The results achieved in this paper represent a step in the right direction but they are by no means final. When we say that the averaged $\bar{P}_{h s}\left(q_{N}\right)$ is the "best" approximation to $P\left(q_{N}\right)$ we do not mean that one cannot do better. In fact, further improvements are always achievable by choosing a broader family of trial distributions. In the study of fluids we saw that these improvements are not just possible, they are necessary. We argued that the next important improvement of the ME method as a calculational tool for fluids should be in the direction of developing a theory of local fluctuations. This would lead to a systematic method for the determination of the effective number of particles $N_{\text {eff }}$ that are locally relevant.

We conclude, therefore, that the ME approach to the theory of fluids is already a significant improvement over the Bogoliuvob variational approach. With only moderate further developments we can realistically expect it to surpass even the best perturbative methods developed to date.

\section{Acknowledgments}

The authors acknowledge R. Scheicher and C.-W. Hong for their valuable assistance and advice with the numerical calculations. 


\section{References}

[1] A. Caticha, "Relative Entropy and Inductive Inference," in these proceedings.

[2] C.-Y. Tseng and A. Caticha, "Maximum Entropy approach to a Mean Field Theory for Fluids," in Bayesian Inference and Maximum Entropy Methods in Science and Engineering, ed. C. J. Williams, AIP Conf. Proc. 659, 73 (2003), online at arXiv.org/abs/cond-mat/0212198.

[3] J. A. Barker and D. Henderson, Rev. Mod. Phys. 48, 587 (1976).

[4] J. P. Hansen and I. R. McDonald, Theory of Simple Liquids (Acad. Press, 1986).

[5] V. I. Kalikmanov, Statistical Physics of Fluids (Springer, 2002).

[6] G. A. Mansoori, F. B. Canfield, J. Chem. Phys. 51, 4958 (1969).

[7] J. D. Weeks, D. Chandler and H. C. Andersen, J. Chem. Phys. 54, 5237 (1971) and Science 220, 797 (1983).

[8] P. Germain and S. Amokrane, Phys. Rev. E65, 031109-1(2002).

[9] A. Caticha, "Maximum Entropy, Fluctuations and Priors," in Bayesian Inference and Maximum Entropy Methods in Science and Engineering, ed. by A. Mohammad-Djafari, AIP Conf. Proc. 568, 94 (2001), online at arXiv.org/abs/math-ph/0008017.

[10] L. Verlet, Phys. Rev. 165, 201 (1968).

[11] J. K.Percus and G. J. Yevick, Phys. Rev. 110, 1 (1958).

[12] M. S. Wertheim, Phys. Rev. Lett. 10, 321 (1963) and J. Math. Phys. 5, 643 (1964); E. Thiele, J.Chem. Phys. 39,474 (1963).

[13] H. Reiss, H. L. Frisch, and J. L. Lebowitz, J. Chem. Phys. 31, 369 (1959).

[14] G. J. Throop and R. J. Bearman, J. Chem. Phys. 42, 2408 (1965). 